\title{
ON THE ALGEBRAIC INDEPENDENCE OF THE VALUES OF SOME E-FUNCTIONS RELATED TO KUMMER'S FUNCTIONS
}

\section{KEIJO VÄ̈̈NÄNEN}

Let $Q$ denote the field of rational numbers, $C$ the field of complex numbers and $C(z)$ the field of rational functions of $z$ with coefficients in $C$.

1. In this paper we apply Sidlovskiǔ's [3] general theorem on the algebraic independence of the values of $E$-functions.

We consider the functions

$$
u_{i, j}(z, v, \mu)=\frac{1}{i ! j !} \frac{\partial^{i+j}}{\partial v^{i} \partial \mu^{j}} K_{v, \mu}(z)
$$

where $K_{v, \mu}(z)$ is the Kummer function

$$
\begin{gathered}
K_{v, \mu}(z)=\sum_{n=0}^{\infty}(\mu(\mu+1) \ldots(\mu+n-1) / n ! v(\nu+1) \ldots(v+n-1)) z^{n}, \\
\mu, v \neq 0,-1,-2, \ldots
\end{gathered}
$$

In [5] some theorems are proved concerning the algebraic independence of the values of $u_{i, 0}(z, v, \mu), u_{i, 0}^{\prime}(z, v, \mu)$ and $u_{0, j}(z, v, \mu), u_{0, j}^{\prime}(z, v, \mu)$. Now the following theorems will be proved.

Th e o r e m 1. Let $v_{k}, \mu_{k}(k=1,2, \ldots, n)$ be rational numbers which satisfy the conditions

$$
\begin{gathered}
v_{k} \neq 0,-1,-2, \ldots, \quad \mu_{k}, \mu_{k}-v_{k} \neq 0, \pm 1, \pm 2, \ldots \\
(k=1,2, \ldots, n) \\
2 \mu_{k}-v_{k}-\left(2 \mu_{r}-v_{v}\right) \pm\left(v_{k} \pm v_{r}\right) \neq 0, \pm 2, \pm 4, \ldots \\
(1 \leqq k<r \leqq n)
\end{gathered}
$$


and let $\alpha \neq 0$ be an algebraic number. Then the numbers of each of the two sets

$$
\begin{gathered}
\left\{u_{0, j}\left(\alpha, v_{k}, \mu_{k}\right), u_{0, j}^{\prime}\left(\alpha, v_{k}, \mu_{k}\right), e^{\alpha}\right\} \\
(j=0,1, \ldots, m ; k=1,2, \ldots, n) \\
\left\{u_{i, 0}\left(\alpha, v_{k}, \mu_{k}\right), u_{i, 0}^{\prime}\left(\alpha, v_{k}, \mu_{k}\right), e^{\alpha}\right\} \\
(i=0,1, \ldots, l ; k=1,2, \ldots, n)
\end{gathered}
$$

are algebraically independent over $Q$.

The orem 2. Let the conditions of Theorem 1 be satisfied. Further, let

$$
v_{k} \neq 1,2, \ldots \quad(k=1,2, \ldots, n) .
$$

Then the $2(l+1)(m+1) n+1$ numbers

$$
\begin{gathered}
u_{i, j}\left(\alpha, v_{k}, \mu_{k}\right), u_{i, j}^{\prime}\left(\alpha, v_{k}, \mu_{k}\right), e^{\alpha} \\
(i=0,1, \ldots, l ; j=0,1, \ldots, m ; k=1,2, \ldots, n)
\end{gathered}
$$

are algebraically independent over $Q$.

It should be noted that by using our theorems and the procedure suggested by Mahler (see [2] p. 172) we could now obtain some transcendental expressions that involve values at rational points of the Gamma function and of its derivatives.

2. In order to prove our theorems we shall first consider the functions

$$
\begin{gathered}
u_{0, j, k}(z)=u_{0, j}\left(z, v_{k}, \mu_{k}\right), \quad u_{0, j, k}^{\prime}(z)=u_{0, j}^{\prime}\left(z, v_{k}, \mu_{k}\right) \\
(j=0,1, \ldots, m ; k=1,2, \ldots, n) .
\end{gathered}
$$

Since the Kummer function $K_{v, \mu}(z)$ satisfies the differential equation

$$
u^{\prime \prime}+\left(\frac{v}{z}-1\right) u^{\prime}-\frac{\mu}{z} u=0,
$$

it follows that the functions $u_{0, j, k}(z) \quad(j=0,1, \ldots, p ; k=1,2, \ldots, n)$ satisfy the following system of differential equations:

$$
\begin{gathered}
u_{0, j, k}^{\prime \prime}+\left(\frac{v_{k}}{z}-1\right) u_{0, j, k}^{\prime}-\frac{\mu_{k}}{z} u_{0, j, k}-\frac{1}{z} u_{0, j-1, k}=0, \quad u_{0,-1, k} \equiv 0 \\
(j=0,1, \ldots, p ; k=1,2, \ldots, n) .
\end{gathered}
$$

Thus if $P$ is a polynomial in $z, u_{0, j, k}, u_{0, j, k}^{\prime}(j=0,1, \ldots, p$; $k=1,2, \ldots, n), e^{\gamma z}, \gamma \neq 0, \gamma \in Q$, then $z P^{\prime}$ is also a polynomial in the same variables. By considering these polynomials in the same way as on pp. 5-6 of [4] we obtain the following lemma. 
$\mathrm{L}$ e $\mathrm{mma} 1$. Let us assume that we have an irreducible polynomial $P$ in $z, u_{0, j, k}, u_{0, j, k}^{\prime}(j=0,1, \ldots, m ; k=1,2, \ldots, n), e^{\gamma z}$ satisfying

$$
P=\sum_{i=0}^{h} P_{i} e^{i \gamma / z}=0 \text {, }
$$

where $P_{i}(i=0,1, \ldots, h)$ are polynomials in $z, u_{0, j, k}, u_{0, j, k}^{\prime}(j=$ $0,1, \ldots, m ; k=1,2, \ldots, n)$ such that $P_{h} \neq 0$. If the functions $u_{0, j, k}$, $u_{0, j, k}^{\prime}(j=0,1, \ldots, m ; k=1,2, \ldots, n)$ are algebraically independent over $C(z)$, then $P$ must satisfy the equation

$$
z P^{\prime}=(a z+b) P, \quad a, b \in Q,
$$

identically in $z, u_{0, j, k}, u_{0, j, k}^{\prime}(j=0,1, \ldots, m ; k=1,2, \ldots, n), \quad e^{\gamma z}$, and must itself be of the form

$$
P=P_{h} e^{h \gamma s}+P_{0}, \quad h \geqq 1,
$$

where the polynomials $P_{h}$ and $P_{0}$ are homogeneous with respect to $u_{0, j, k}$, $u_{0, j, k}^{\prime}(j=0,1, \ldots, m ; k=1,2, \ldots, n)$ and one of them is a polynomial in $z$ alone.

This lemma can be used in the proof of Lemma 2.

$\mathrm{L}$ e $\mathrm{m} \mathrm{m}$ a 2. Let us assume that $v_{k}, \mu_{k}(k=1,2, \ldots, n)$ satisfy the conditions of Theorem 1 and let $\gamma \neq 0$ be a rational number. Then the functions (3) and $e^{\gamma z}$ are algebraically independent over $C(z)$.

The proof will be performed by induction with respect to $m$.

3. Let $m=0$. The algebraic independence of the functions $u_{0,0, k}$, $u_{0,0, k}^{\prime}(k=1,2, \ldots, n)$ follows from Lemma 7 of [1]. If these functions and $e^{\gamma z}$ were algebraically dependent over $C(z)$, we would then have an equation of the form (6) (with $m=0$ ). By Lemma 1, $P$ satisfies (7) and is of the form (8), where $P_{h}=c_{h} z^{b}$ or $P_{0}=c_{0} z^{b}, c_{0} \neq 0$ and $c_{h} \neq 0$.

If $P_{h}=c_{h} z^{b}$, then

$$
P=c_{h} z^{b} e^{h \gamma z}+P_{0}=0 .
$$

Let $r$ be the greatest index such that $u_{0,0, r}$ or $u_{0,0, r}^{\prime}$ occurs in $P_{0}$, and let $s$ be the degree of $P_{0}$ with respect to $u_{0,0, k}, u_{0,0, k}^{\prime}(k=1,2, \ldots, n)$. When we present $P_{0}$ in the form

$$
P_{0}=\sum_{i=0}^{p} \sum_{j=0}^{i} P_{i j} u_{0,0, r}^{i-j} u_{0,0, r}^{\prime j}, \quad p \geqq 1,
$$

where at least one $P_{p j} \neq 0$, and denote $B_{k}=e^{-z / 2} u_{0,0, k}$, we obtain by $(9)$

$$
P=c_{h} z^{b} e^{h \gamma z}+e^{s z / 2} \sum_{i=0}^{p} \sum_{j=0}^{i} P_{i j}^{*} B_{r}^{i-j}\left(B_{r} / 2+B_{r}^{\prime}\right)^{j}=0,
$$


where $P_{i j}^{*}$ are polynomials in $z, B_{k}, B_{k}^{\prime}(k=1,2, \ldots, r-1)$ such that at least one $P_{p j}^{* \neq} \neq 0$. The equation (10) implies that the functions $e^{z / 2}$, $B_{k}, \quad B_{k}^{\prime} \quad(k=1,2, \ldots, r)$ are algebraically dependent over $C(z)$. This contradicts the proof of Lemma 7 in [1], by which these functions are algebraically independent.

If $P_{0}=c_{0} z^{b}$ we obtain the same contradiction.

4. For all $k=1,2, \ldots, n$ in the following let the notations $u_{k}(m)$ and $u_{k}^{\prime}(m)$ denote the functions $u_{0, j, k}$ and $u_{0, j, k}^{\prime}(j=0,1, \ldots, m)$ respectively.

Now let us assume that the functions $u_{k}(m), u_{k}^{\prime}(m) \quad(k=1,2, \ldots, n)$ and $e^{\gamma z}$ are algebraically independent over $C(z)$ for some $m=0,1, \ldots$. We shall prove that this implies the algebraic independence over $C(z)$ of the functions $u_{k}(m+1), u_{k}^{\prime}(m+1)(k=1,2, \ldots, n)$ and $e^{\gamma z}$.

Let the functions $u_{k}(m+1), u_{k}^{\prime}(m+1)(k=1,2, \ldots, n)$ and $e^{\gamma z}$ be algebraically dependent over $C(z)$. Then there exists an integer $q \in\{1,2, \ldots, n\}$ such that the functions

$$
\begin{gathered}
u_{k}(m), u_{k}^{\prime}(m), \quad u_{0, m+1, i}, u_{0, m+1, i}^{\prime} \\
(k=1,2, \ldots, n ; i=1,2, \ldots, q-1)
\end{gathered}
$$

and $e^{\gamma z}$ are algebraically independent over $C(z)$, while the functions (11), $u_{0, m+1, q}, \quad u_{0, m+1, q}^{\prime}$ and $e^{\gamma z}$ are algebraically dependent over $C(z)$. We shall prove that this is impossible. The proof is divided into three steps, called A, B and C, which are analogous to the corresponding steps in $[5]$.

5. Step A. We define

$$
t=u_{0,0, q} u_{0, m+1, q}^{\prime}-u_{0,0, q}^{\prime} u_{0, m+1, q}
$$

and prove that the functions (11) and $t$ are algebraically independent over $C(z)$. If this were not true then there would exist an irreducible polynomial $P$ in $z,(11)$ and $t$ such that

$$
P=\sum_{i=0}^{h} P_{i} t^{i}=0,
$$

where $P_{i} \quad(i=0,1, \ldots, h)$ are polynomials in $z$ and (11) such that $P_{h} \not \equiv 0$. From our assumptions it follows that $h \geqq 1$.

By (5) z $P^{\prime}$ is also a polynomial in the same variables as $P$. Analogously to p. 97 of [5] we obtain that

$$
z P^{\prime}=(a z+b) P, \quad a, b \in Q,
$$

identically in $z,(11)$ and $t$. 
The identity (13) holds even when we replace the functions (11) by the corresponding functions of any other solution $\bar{u}_{k}(m+1), \quad \bar{u}_{k}^{\prime}(m+1)$ $(k=1,2, \ldots, n)$ of (5) (with $p=m+1$ ) and $t$ by $\bar{t}=\bar{u}_{0,0, q} \bar{u}_{0, m+1, q}^{\prime}-$ $\bar{u}_{0,0, q}^{\prime} \bar{u}_{0, m+1, q}$. Thus we obtain, by analogy with pp. 97-98 of [5], that $a=h=1$ and $P$ is of the form

$$
P=c_{0} z^{b+v_{q}} t+P_{0},
$$

where $P_{0}$ is a homogeneous polynomial of the second degree with respect to (11).

When we now choose $\bar{u}_{k}(m+1)=u_{k}(m+1)(k=1,2, \ldots, n, k \neq q)$ and $\bar{u}_{q}(m+1)$ in the same manner as $u(k+1)$ in [5], we obtain for $P$, entirely analogously to pp. $98-99$ of [5], the form

$$
P=\sum_{i=0}^{s} a_{i} \sum_{j=0}^{s-i}\left(u_{0, j, q} u_{0, m+1-i-j, q}^{\prime}-u_{0, j, q}^{\prime} u_{0, m+1-i-j, q}\right)+A_{s},
$$

where $s=[m / 2], \quad a_{0}=c_{0} z^{b+v} q, \quad a_{i}(i=1, \ldots, s)$ are polynomials in $z$ and $A_{s}$ is a homogeneous polynomial of the second degree with respect to $u_{k}(m+1), \quad u_{k}^{\prime}(m+1) \quad(k=1,2, \ldots, q-1), \quad u_{k}(m), \quad u_{k}^{\prime}(m)$ $(k=q+1, \ldots, n)$ and $u_{0, j, q}, u_{0, j, q}^{\prime}(j=0,1, \ldots, m-s)$.

It can be proved in an exactly analogous way to pp. 99-101 of [5] that polynomial of the form (15) cannot satisfy the differential equation (13) (where $a=1$ ). Thus the functions (11) and $t$ are algebraically independent over $C(z)$.

6. The algebraic independence over $C(z)$ of the functions (11), $u_{0, m+1, q}$ and $u_{0, m+1, q}^{\prime}$ (step B) can now be proved by using a similar deduction as in step $\mathrm{B}$ of [5]. Only certain formal changes need to be made, so that the proof is not repeated here. The same is true in the proof of the algebraic independence over $C(z)$ of the functions (11), $u_{0, m+1, q} u_{0, m+1, q}^{\prime}$ and $e^{\gamma z}$ (step $\left.\mathrm{C}\right)$, which can be performed analogously to step $\mathrm{C}$ of [5]. Thus Lemma 2 is true.

7. The last part of this paper is mainly concerned with the functions

$$
\begin{aligned}
& u_{i, j, k}(z)=u_{i, j}\left(z, v_{k}, \mu_{k}\right), \quad u_{i, j, k}^{\prime}(z)=u_{i, j}^{\prime}\left(z, v_{k}, \mu_{k}\right) \\
& (i=0,1, \ldots, l ; j=0,1, \ldots, m ; k=1,2, \ldots, n) .
\end{aligned}
$$

By (5) these functions satisfy

$$
\begin{aligned}
& \text { (17) } u_{i, j, k}^{\prime \prime}+\left(\frac{v_{k}}{z}-1\right) u_{i, j, k}^{\prime}-\frac{\mu_{k}}{z} u_{i, j, k}-\frac{1}{z} u_{i, j-1, k}+\frac{1}{z} u_{i-1, j, k}^{\prime}=0, \\
& u_{-1, j, k} \equiv u_{i,-1, k} \equiv 0 \quad(i=0,1, \ldots, l ; j=0,1, \ldots, m ; k=1,2, \ldots, n) .
\end{aligned}
$$

We prove the following lemma. 
L e $\mathrm{m} \mathrm{m} \mathrm{a} 3$. Let $v_{k}, \quad \mu_{k}(k=1,2, \ldots, n)$ satisfy the conditions of Theorem 2 and let $\gamma \neq 0$ be a rational number. Then the functions (16) and $e^{\gamma z}$ are algebraically independent over $C(z)$.

We shall prove this by induction with respect to $l$. If $l=0$, then Lemma 3 follows from Lemma 2.

Before continuing we should adopt some simplifying notations. For all $k=1,2, \ldots, n$ let the notations $u_{k}(l, m)$ and $u_{k}^{\prime}(l, m)$ denote respectively the functions $u_{i, j, k}$ and $u_{i, j, k}^{\prime} \quad(i=0,1, \ldots, l$; $j=0,1, \ldots, m)$.

Now suppose that Lemma 3 is true for some $l=0,1, \ldots$. This means that the functions $u_{k}(l, m), \quad u_{k}^{\prime}(l, m) \quad(k=1,2, \ldots, n)$ and $e^{\gamma z}$ are algebraically independent over $C(z)$. By using this assumption we shall prove the algebraic independence over $C(z)$ of the functions $u_{k}(l+1, m)$, $u_{k}^{\prime}(l+1, m) \quad(k=1,2, \ldots, n)$ and $e^{\gamma z}$.

If these functions were algebraically dependent over $C(z)$, then, by the induction hypothesis, there exists an integer $p$ such that the functions

$$
\begin{gathered}
u_{k}(l, m), u_{k}^{\prime}(l, m), u_{l+1, j, k}, u_{l+1, j, k}^{\prime}, e^{\gamma z} \\
(j=0,1, \ldots, p-1 ; k=1,2, \ldots, n)
\end{gathered}
$$

are algebraically independent over $C(z)$, but these functions (18) and $u_{l+1, p, k}, \quad u_{l+1, p, k}^{\prime} \quad(k=1,2, \ldots, n)$ are algebraically dependent over $C(z)$. This yields the existence of an integer $q$ such that the functions (18) and $u_{l+1, p, i}, \quad u_{l+1, p, i}^{\prime}(i=1,2, \ldots, q-1)$ are algebraically independent over $C(z)$, but these functions and $u_{l+1, p, q}, \quad u_{l+1, p, q}^{\prime}$ are algebraically dependent over $C(z)$.

In the following let assumption $\mathrm{A}$ denote the algebraic independence of the functions (18) and $u_{l+1, p, i}, u_{l+1, p, i}^{\prime}(i=1,2, \ldots, q-1)$. Further let $\{u\}$ denote the following set of functions,

$$
\begin{gathered}
\left\{u_{k}(l, m), u_{k}^{\prime}(l, m), u_{l+1, j, k}, u_{l+1, j, k}^{\prime}, u_{l+1, p, i}, u_{l+1, p, i}^{\prime}\right\} \\
(j=0,1, \ldots, p-1 ; i=1,2, \ldots, q-1 ; k=1,2, \ldots, n, k \neq q) .
\end{gathered}
$$

8. We denote

$$
t=u_{0,0, q} u_{l+1, p, q}^{\prime}-u_{0,0, q}^{\prime} u_{l+1, p, q}
$$

and prove that the functions

$$
\{u\}, u_{q}(l, m), u_{q}^{\prime}(l, m), \quad u_{l+1, j, q}, u_{l+1, j, q}^{\prime} \quad(j=0,1, \ldots, p-1)
$$

and $t$ are algebraically independent over $C(z)$. If this were not true, then we would have an equation

$$
P=\sum_{i=0}^{h} P_{i} t^{i}=0,
$$


where $P$ is an irreducible polynomial in $z,(19)$ and $t$, and $P_{i}$ $(i=0,1, \ldots, h)$ are polynomials in $z$ and $(19)$ such that $P_{h} \neq 0$. By the assumption $\mathrm{A} h \geqq 1$.

As on pp. 97-98 of [5] we can again deduce that the polynomial $P$ satisfies

$$
z P^{\prime}=(z+b) P, \quad b \in Q,
$$

identically in $z,(19)$ and $t$, and is of the form

$$
P=c_{0} z^{b+v_{q}} t+P_{0}=0, \quad c_{0} \neq 0,
$$

where $P_{0}$ is a homogeneous polynomial of the second degree with respect to (19).

Since (21) is an identity, it follows that (21) holds even when we replace the functions (19) by any other solution $\{\bar{u}\}, \bar{u}_{q}(l, m), \bar{u}_{q}^{\prime}(l, m), \bar{u}_{l+1, j, q}$, $\bar{u}_{l+1, j, q}^{\prime}(j=0,1, \ldots, p-1)$ of $(17)$ and $t$ by

$$
t=\bar{u}_{0,0, q} \bar{u}_{l+1, p, q}^{\prime}-\bar{u}_{0,0, q}^{\prime} \bar{u}_{l+1, p, q} \text {. }
$$

This fact will be used in the following deduction.

9. Next we prove that a contradiction follows if $p>0$. For this we present $P_{0}$ in the form

$$
\begin{aligned}
P_{0}= & A_{1}+P_{10} u_{l+1, p-1, q}+P_{01} u_{l+1, p-1, q}^{\prime}+P_{20} u_{l+1, p-1, q}^{2} \\
& +P_{11} u_{l+1, p-1, q} u_{l+1, p-1, q}^{\prime}+P_{02} u_{l+1, p-1, q}^{\prime 2},
\end{aligned}
$$

where $A_{1} ; P_{10}, P_{01}$ and $P_{20}, P_{11}, P_{02}$ are homogeneous polynomials of degree two; one and zero, respectively, with respect to $\{u\}, u_{q}(l, m)$, $u_{q}^{\prime}(l, m), \quad u_{l+1, j, q}, \quad u_{l+1, j, q}^{\prime}(j=0,1, \ldots, p-2)$.

Replace now in (21) the functions $\{u\}, u_{q}(l, m), u_{l+1, j, q}(j=0,1, \ldots, p)$ by the functions $\{\bar{u}\}=\{u\}, \quad \bar{u}_{q}(l, m)=u_{q}(l, m), \quad \bar{u}_{l+1, j, q}=u_{l+1, j, q}$ $(j=0,1, \ldots, p-2), \bar{u}_{l+1, j, q}=u_{l+1, j, q}+\sigma u_{0, j-p+1, q}(j=p-1, p)$ with an arbitrary constant $\sigma$. We then integrate (21), differentiate the result with respect to $\sigma$ and put $\sigma=0$. This yields the equation

$$
\begin{gathered}
c_{0} z^{b+v_{q}}\left(u_{0,0, q} u_{0,1, q}^{\prime}-u_{0,0, q}^{\prime} u_{0,1, q}\right)+P_{10} u_{0,0, q}+P_{01} u_{0,0, q}^{\prime} \\
+2 P_{20} u_{0,0, q} u_{l+1, p-1, q}+P_{11}\left(u_{0,0, q}^{\prime} u_{l+1, p-1, q}+u_{0,0, q} u_{l+1, p-1, q}^{\prime}\right) \\
+2 P_{02} u_{0,0, q}^{\prime} u_{l+1, p-1, q}^{\prime}=c^{\prime}(0) z^{b} e^{z} .
\end{gathered}
$$

From this it follows by the assumption $\mathrm{A}$ that

$$
P=\sum_{h=0}^{1} a_{h} \sum_{j=0}^{1-h}\left(u_{0, j, q} u_{l+1, p-h-j, q}^{\prime}-u_{0, j, q}^{\prime} u_{l+1, p-h-j, q}\right)+A_{1},
$$

where $a_{0}=c_{0} z^{b+v_{q}} \neq 0$ and $a_{1}$ is a polynomial in $z$. 
Let us assume that we have for some $s<p$

$$
P=\sum_{h=0}^{s} a_{h} \sum_{j=0}^{s-h}\left(u_{0, j, q} u_{l+1, p-h-j, q}^{\prime}-u_{0, j, q}^{\prime} u_{l+1, p-h-j, q}\right)+A_{s}
$$

where $a_{h}$ are polynomials in $z, a_{0}=c_{0} z^{b+v_{q}} \neq 0$, and

$$
\begin{aligned}
A_{s}= & A_{s+1}+P_{10} u_{l+1, p-s-1, q}+P_{01} u_{l+1, p-s-1, q}^{\prime}+P_{20} u_{l+1, p-s-1, q}^{2} \\
& +P_{11} u_{l+1, p-s-1, q} u_{l+1, p-s-1, q}^{\prime}+P_{02} u_{l+1, p-s-1, q}^{\prime 2} .
\end{aligned}
$$

Now let $\{\bar{u}\}=\{u\}, \quad \bar{u}_{q}(l, m)=u_{q}(l, m), \quad \bar{u}_{l+1, j, q}=u_{l+1, j, q}, \quad(j=$ $0,1, \ldots, p-s-2), \bar{u}_{l+1, j, q}=u_{l+1, j, q}+\sigma u_{0, j-p+s+1, q}(j=p-s-1, \ldots, p)$. After the same steps as before we obtain for $P$ the form

$$
P=\sum_{h=0}^{s+1} a_{h} \sum_{j=0}^{s+1-h}\left(u_{0, j, q} u_{l+1, p-h-j, q}^{\prime}-u_{0, j, q}^{\prime} u_{l+1, p-h-j, q}\right)+A_{s+1} .
$$

Thus it follows that

$$
P=\sum_{h=0}^{p} a_{h} \sum_{j=0}^{p-h}\left(u_{0, j, q} u_{l+1, p-h-j, q}^{\prime}-u_{0, j, q}^{\prime} u_{l+1, p-h-j, q}\right)+A,
$$

where $a_{h}$ are polynomials in $z$, in particular $a_{0}=c_{0} z^{b+v_{q}} \neq 0$, and $A$ is a polynomial in $z,\{u\}, u_{q}(l, m), u_{q}^{\prime}(l, m)$ of the second degree with respect to $\{u\}, u_{q}(l, m), u_{q}^{\prime}(l, m)$.

We now use in (21) the functions $\{\bar{u}\}=\{u\}, \quad \bar{u}_{q}(l+1, p-1)=$ $u_{q}(l+1, p-1), \quad \bar{u}_{i, j, q}=u_{i, j, q}+\sigma u_{i, j-p, q} \quad(i=0,1, \ldots, l+1 ; \quad j=$ $p, \ldots, m)$. By integrating (21) we then obtain

$$
\begin{gathered}
\sum_{h=0}^{p} a_{h} \sum_{j=0}^{p-h}\left(u_{0, j, q} u_{l+1, p-h-j, q}^{\prime}-u_{0, j, q}^{\prime} u_{l+1, p-h-j, q}\right) \\
+2 a_{0} \sigma\left(u_{0,0, q} u_{l+1,0, q}^{\prime}-u_{0,0, q}^{\prime} u_{l+1,0, q}\right)+\bar{A}=c(\sigma) z^{b} e^{z} .
\end{gathered}
$$

This gives

$$
2 a_{0}\left(u_{0,0, q} u_{l+1,0, q}^{\prime}-u_{0,0, q}^{\prime} u_{l+1,0, q}\right)+\left.\frac{\partial \bar{A}}{\partial \sigma}\right|_{\sigma=0}=c^{\prime}(0) z^{b} e^{z},
$$

which is impossible. Thus we are left with the case $p=0$.

10. If $l=0$, then we obtain a contradiction in an entirely analogous way to pp. $107-108$ of [5].

If $l=1$ and none of the functions $u_{1, s, q}, u_{1, s, q}^{\prime}(s \geq 1)$ occur in $P$, then a contradiction arises as on p. 107 in [5]. If $s \geq 1$ is the greatest integer such that at least one of the functions $u_{1, s, q}$ or $u_{1, s, q}^{\prime}$ occurs in $P$, then we obtain, as in Section 9 , for $P$ the form 


$$
P=c_{0} z^{b+v_{q}} t+\sum_{h=0}^{s-1} a_{h} \sum_{j=0}^{s-1-h}\left(u_{0, j, q} u_{1, s-h-j, q}^{\prime}-u_{0, j, q}^{\prime} u_{1, s-h-j, q}\right)+A_{s-1},
$$

where $a_{h}$ are polynomials in $z, a_{0} \neq 0$, and $A_{s-1}$ is of the form

$$
\begin{aligned}
A_{s-1}= & A+P_{10} u_{1,0, q}+P_{01} u_{1,0, q}^{\prime}+P_{20} u_{1,0, q}^{2} \\
& +P_{11} u_{1,0, q} u_{1,0, q}^{\prime}+P_{02} u_{1,0, q}^{\prime 2},
\end{aligned}
$$

where $A ; P_{10}, P_{01}$ and $P_{20}, P_{11}, P_{02}$ are homogeneous polynomials of degree two; one and zero, respectively, with respect to $\{u\}, u_{q}(0, m)$, $u_{q}^{\prime}(0, m)$. We now replace in $(21) \quad\{u\}$ and $u_{q}(2, m)$ by $\{\bar{u}\}=\{u\}$, $\bar{u}_{q}(0, m)=u_{q}(0, m), \quad \bar{u}_{i, j, q}=u_{i, j, q}+\sigma u_{i-1, j, q} \quad(i=1,2, ; \quad j=$ $0,1, \ldots, m)$. By integrating $(21)$, differentiating the result with respect to $\sigma$ and putting $\sigma=0$ we obtain

$$
\begin{gathered}
c_{0} z^{b+v}\left(u_{0,0, q} u_{1,0, q}^{\prime}-u_{0,0, q}^{\prime} u_{1,0, q}\right) \\
+\sum_{h=0}^{s-1} a_{h} \sum_{j=0}^{s-1-h}\left(u_{0, j, q} u_{0, s-h-j, q}^{\prime}-u_{0, j, q}^{\prime} u_{0, s-h-j, q}\right) \\
+P_{10} u_{0,0, q}+P_{01} u_{0,0, q}^{\prime}+2 P_{20} u_{0,0, q} u_{1,0, q} \\
+P_{11}\left(u_{0,0, q} u_{1,0, q}^{\prime}+u_{0,0, q}^{\prime} u_{1,0, q}\right) \\
+2 P_{02} u_{0,0, q}^{\prime} u_{1,0, q}^{\prime}=c^{\prime}(0) z^{b} e^{z} .
\end{gathered}
$$

Since $c_{0} \neq 0$, the assumption A implies a contradiction.

If $l>1$, denote $g=[l / 2]$. If none of the functions $u_{i, j, q}, u_{i, j, q}^{\prime}$ $(l+1-g \leqq i \leqq l ; j=1,2, \ldots, m)$ occur in $P$ we obtain a contradiction in a completely analogous way to p. 106 of [5]. Thus some of these functions must occur in $P$. Let either $u_{r, s, q}$ or $u_{r, s, q}^{\prime}$ be the one having the greatest $i$ and greatest $j$ with this $i$. First we can easily deduce that then $P$ must be of the form

$$
\begin{aligned}
& P=\sum_{h=0}^{l-r} b_{h} \sum_{j=0}^{l-r-h}\left(u_{j, 0, q} u_{l+1-h-j, 0, q}^{\prime}-u_{j, 0, q}^{\prime} u_{l+1-h-j, 0, q}\right) \\
& +\sum_{h=0}^{s-1} a_{h} \sum_{j=0}^{s-1-h}\left(u_{0, j, q} u_{r, s-h-j, q}^{\prime}-u_{0, j, q}^{\prime} u_{r, s-h-j, q}\right)+A,
\end{aligned}
$$

where $b_{h}$ and $a_{h}$ are polynomials in $z$ such that $b_{0}=c_{0} z^{b+v_{q}} \neq 0$, $a_{0} \neq 0$, and a polynomial $A$ can be presented in the typical form

$$
\begin{aligned}
A= & B+P_{10} u_{r, 0, q}+P_{01} u_{r, 0, q}^{\prime}+P_{20} u_{r, 0, q}^{2} \\
& +P_{11} u_{r, 0, q} u_{r, 0, q}^{\prime}+P_{02} u_{r, 0, q}^{\prime 2} .
\end{aligned}
$$

We now use in (21) the solution $\{\bar{u}\}=\{u\}, \bar{u}_{q}(r-1, m)=u_{q}(r-1, n)$, $\bar{u}_{i, j, q}=u_{i, j, q}+\sigma u_{i-r, j, q} \quad(i=r, \ldots, l+1 ; \quad j=0,1, \ldots, m)$. When 
we then integrate (21), differentiate the result with respect to $\sigma$ and put $\sigma=0$, we obtain an equation

$$
\begin{gathered}
\sum_{h=0}^{l-r} b_{h}\left(u_{0,0, q} u_{l+1-h-r, 0, q}^{\prime}-u_{0,0, q}^{\prime} u_{l+1-h-r, 0, q}\right) \\
+\sum_{h=0}^{s-1} a_{h}\left(u_{0,0, q} u_{0, s-h, q}^{\prime}-u_{0,0, q}^{\prime} u_{0, s-h, q}\right)+P_{10} u_{0,0, q}+P_{01} u_{0,0, q}^{\prime} \\
+2 P_{20} u_{0,0, q} u_{r, 0, q}+P_{11}\left(u_{0,0, q} u_{r, 0, q}^{\prime}+u_{0,0, q}^{\prime} u_{r, 0, q}\right) \\
+2 P_{02} u_{0,0, q}^{\prime} u_{r, 0, q}^{\prime}=c^{\prime}(0) z^{b} e^{z} .
\end{gathered}
$$

Since $r \geqq l+1-g$, we have $r>l+1-r$. Thus the assumption A implies that $P_{20} \equiv P_{11} \equiv P_{02} \equiv c^{\prime}(0)=0$, and it also follows from assumption A that $P$ has the form

$$
P=\sum_{h=0}^{l+1-r} b_{h} P_{h}+\sum_{h=0}^{s-1} a_{h} Q_{h}+B,
$$

in which we have denoted

$$
\begin{gathered}
P_{h}=\sum_{j=0}^{l+1-r-h}\left(u_{j, 0, q} u_{l+1-h-j, 0, q}^{\prime}-u_{j, 0, q}^{\prime} u_{l+1-h-j, 0, q}\right), \\
Q_{h}=\sum_{j=0}^{s-h}\left(u_{0, j, q} u_{r, s-h-j, q}^{\prime}-u_{0, j, q}^{\prime} u_{r, s-h-j, q}\right) .
\end{gathered}
$$

By (21)

$$
\begin{gathered}
\sum_{h=0}^{l+1-r}\left(z b_{h}^{\prime}-\left(b+v_{q}\right) b_{h}\right) P_{h}-\sum_{h=1}^{l+1-r} b_{h-1} P_{h}-\sum_{h=0}^{l+1-r} b_{h} u_{l+1-r-h, 0, q} u_{r-1,0, q}^{\prime} \\
+\sum_{h=0}^{s-1}\left(z a_{h}^{\prime}-\left(b+v_{q}\right) a_{h}\right) Q_{h}-\sum_{h=0}^{s-1} a_{h} \sum_{j=0}^{s-h} u_{0, j, q} u_{r-1, s-h-j, q}^{\prime} \\
+z B^{\prime}=(z+b) B .
\end{gathered}
$$

Now $z b_{0}^{\prime}-\left(b+v_{q}\right) b_{0}=0$. Thus it follows by the assumption A that

$$
\begin{gathered}
z a_{h}^{\prime}-\left(b+v_{q}\right) a_{h}=0 \quad(h=0, \ldots, s-1), \\
z b_{h}^{\prime}-\left(b+v_{q}\right) b_{h}-b_{h-1}=0 \quad(h=1, \ldots, l+1-r) .
\end{gathered}
$$

In particular, $z b_{1}^{\prime}-\left(b+v_{q}\right) b_{1}-c_{0} z^{b+v_{q}}=0$. If $b+v_{q}=0$, then we obtain the contradiction $z b_{1}^{\prime}=c_{0}$. If $b+v_{q}>0$, then we have

$$
b_{1}=c_{1} z^{i}+c_{2} z^{i+1}+\ldots, \quad c_{1} \neq 0, \quad b+v_{q} \geqq i>0 .
$$

This gives

$$
\left(i-b-v_{q}\right) c_{1}-\delta_{i, b+v_{q}} c_{0}=0,
$$


which is again impossible. Thus the functions (19) and $t$ are algebraically independent over $C(z)$.

11. In an entirely analogous way to steps $B$ and $C$ of [5] we can now prove the algebraic independence over $C(z)$ of the functions (19), $u_{l+1, p, q}, \quad u_{l+1, p, q}^{\prime}$ and $e^{\gamma z}$.

This implies that the functions $u_{k}(l+1, m), u_{k}^{\prime}(l+1, m)(k=1,2, \ldots, n)$ and $e^{\gamma z}$ are algebraically independent over $C(z)$. In this way we have established the truth of Lemma 3.

It should be noted that if $m=0$, then the assumption $v_{k} \neq 1,2, \ldots$ $(k=1,2, \ldots, n)$ of Lemma 3 is unnecessary. Thus we have proved at the same time the following lemma.

$\mathrm{L}$ e $\mathrm{mm}$ a 4 . Let us assume that $v_{k}, \mu_{k}(k=1,2, \ldots, n)$ satisfy the conditions of Theorem 1 and let $\gamma \neq 0$ be a rational number. Then the functions

$$
u_{i, 0, k}(z), u_{i, 0, k}^{\prime}(z), e^{\gamma z} \quad(i=0,1, \ldots, l ; k=1,2, \ldots, n)
$$

are algebraically independent over $C(z)$.

12. The functions $u_{i, j, k}(z), u_{i, j, k}^{\prime}(z)(i=0,1, \ldots, l ; j=0,1, \ldots, m$; $k=1,2, \ldots, n)$ and $e^{z}$ are $E$-functions and satisfy, by (17), the following system of differential equations:

$$
\begin{gathered}
\left(u_{i, j, k}\right)^{\prime}=u_{i, j, k}^{\prime}, \quad\left(u_{i, j, k}^{\prime}\right)^{\prime}=\left(1-\frac{v_{k}}{z}\right) u_{i, j, k}^{\prime}+\frac{\mu_{k}}{z} u_{i, j, k} \\
+\frac{1}{z} u_{i, j-1, k}-\frac{1}{z} u_{i-1, j, k}^{\prime}=0, \\
u_{-1, j, k} \equiv u_{i,-1, k} \equiv 0 \\
(i=0,1, \ldots, l ; \quad j=0,1, \ldots, m ; k=1,2, \ldots, n), \quad y^{\prime}=y .
\end{gathered}
$$

Thus Šdlovskiî's [3] general theorem on the algebraic independence of the values of $E$-functions together with Lemmas 2 and 4 establishes the truth of Theorem 1. Theorem 2 follows from Šidlovskiřs result and Lemma 3.

\section{References}

[1] BeIOGRIVOV, I. I.: О трансцендентности и алгебраической независимости значений функций куммера. - Sibirsk. Mat. Ž. 12, 1971, 961-982. Translation: On transcendence and algebraic independence of the values of Kummer's functions. - Siberian Math. J. 12, 1971, 690-705.

[2] MamrER, K.: Applications of a theorem by A. B. Shidlovski. - Proc. Roy. Soc. Ser. A $305,1968,149-173$. 
[3] ŠIDLovskǏ̃, A. В.: О трансцендентности и алгебраической независимости значений $E$-функций, связанных любым числом алгебраических уравнений в поле рациональных функций. - Izv. Akad. Nauk SSSR Ser. Mat. 26, 1962, 877-910. Translation: On the transcendence and algebraic independence of values of $E$-functions related by an arbitrary number of algebraic equations over the field of rational functions. - Amer. Math. Soc. Transl. (2) 50, 1966, $141-177$.

[4] V̈̈̈̈ӓnEN, K.: On the transcendence and algebraic independence of the values of certain $E$-functions. - Ann. Acad. Sci. Fenn. Ser. A I 537, 1973, 1 - 16.

[5] - - On the algebraic independence of the values of some $E$-functions. Ann. Acad. Sci. Fenn. Ser. A I 1, 1975, 93-109.

University of Oulu

Department of Mathematics

SF-90100 Oulu 10

Finland

Received 3 February 1975 